\title{
Fig. S13A
}

\# 1: A_Sequence: Nps1 TMS \#1-3 (Q8YX33; 2.A.108 homologue)

\# 2: B Sequence: Nps1 TMS \#4-6,7

\# Matrix: EBLOSUM62

\# Gap_penalty: 8.0

\# Extend_penalty: 2.0

\#

\# Length: 150

\# Identity: $\quad 30 / 150 \quad(20.0 \%)$

\# Similarity: $50 / 150 \quad(33.3 \%)$

\# Gaps: $\quad 54 / 150$ (36.0\%)

\# Score: 99.0

\#

\#

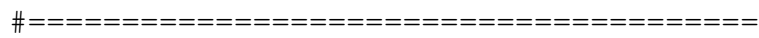

$1(D / E) \times X E$

A_sequence

1 ---------MNWEIVLASFASSLIELVEILGIVIVVGRL-AGWRNALV

B_Sequence

||$|::|:|::||: \quad|::|\quad:| 1 \mid$

1 EQLARDENESGWNWFAVVTTFKGALLDSVEVAIAVVTLGAAQSQWLEAIS

A_Sequence

2

4 (D/E) XXE

B_Sequence

39 GSGAGIALTLLLSLVLGKSLTLI PVNI LR IVAGVLLLLFGQKWTRS IVRY

$|:| \quad|:|:: \quad|\quad:||::|:||:|| \mid$

A_sequence

89 YAGLPKKRKGGGEDSI

$\frac{\text { AMLLMGFGLYWLG----- }}{6}$

95

B_Sequence

96 -AGLNVEWPG---DELAI IWLPLAWGVGMA IAST IWRWRVS LDKPEEAIG

105

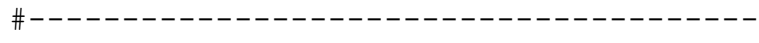

$==========$ FINISHED $============$

Average Quality (AQ)

$20.11+/-7.38$

Standard score (Z): 11.0

Precise score (Z): 10.7 


\section{Fig. S13B}

\# 1: A_Sequence: Nps3 TMS \#1-3 (K9Q6B8; 2.A.108 homologue)

\# 2: B Sequence: Nps3 TMS \#4-6,7

\# Matrix: EBLOSUM62

\# Gap_penalty: 8.0

\# Extend_penalty: 2.0

\#

\# Length: 151

\# Identity: $\quad 30 / 151$ (19.9\%)

\# Similarity: $\quad 47 / 151$ (31.1\%)

\# Gaps: $58 / 151 \quad(38.4 \%)$

\# Score: 96.0

\#

\#

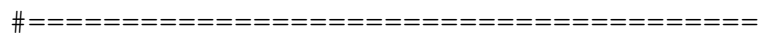
A_Sequence
B_Sequence
1
B_Sequence
1 LETELANTG
MNWEIFLASFVGSLIELVEILGLVLIVGKLAG-WRNA 36
A_sequence
B_Sequence
2
4 (D/E) XXE 3
37 FVGA-GSGIGLTLLASLILGTSLTIIPVDILRIVAGVFLLAFGQKWTRSI 85

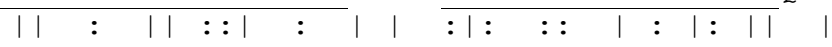
51 AGGASAAAFGLVVVA-FLFRTPLNQVPIKPMKFTAAMLLMGFGIYWLSE-

$1(\mathrm{D} / \mathrm{E}) \times \times \mathrm{E}$

A_Sequence

86 VKYYAGIPKKRKDEEDD

102

B_Sequence

$99-----G F--K I K L P G D D W A I V W L P I V W G C L M A V S A L L L R W Q V G L Q P K E I V$

$\begin{array}{lll}\text { A_Sequence } & 102- & 102 \\ \text { B_Sequence } & 142 \mathrm{~S} & 142\end{array}$

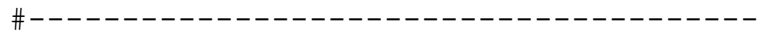

$==========$ FINISHED $============$

Average Quality (AQ) $\quad 21.97$ +/- 7.85

Standard score (Z): 9.0

Precise score (Z): $\mathbf{9 . 4}$ 


\section{Fig. S13C}

\# 1: A_Sequence: Rsp3 TMS \#1-3 (J2KV33; 2.A.108 homologue)

\# 2: B Sequence: Rsp3 TMS \#4-6,7

\# Matrix: EBLOSUM62

\# Gap_penalty: 8.0

\# Extend_penalty: 2.0

\#

\# Length: 140

\# Identity: $\quad 37 / 140(26.4 \%)$

\# Similarity: $\quad 56 / 140(40.0 \%)$

\# Gaps: $30 / 140(21.4 \%)$

\# Score: 84.0

\#

\#

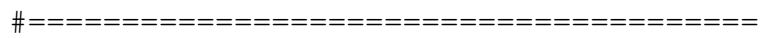

$1(D / E) \times X E$

A_Sequence 1 MTTITSITSTMA--ASFLGSFVEVVEAFTIILAVGVTQSWRPAFIGTGLA 48

B_Sequence 1 --SADRRADFLAGTAAFKAVLLEGVEVVFIVIATGARPGMLP-YAGLGAL 47

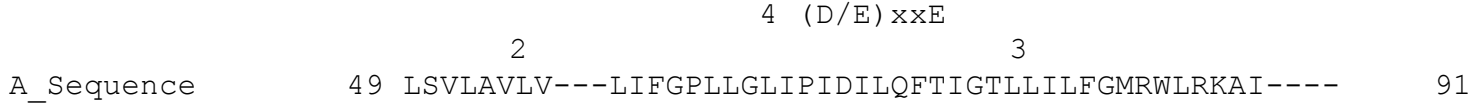

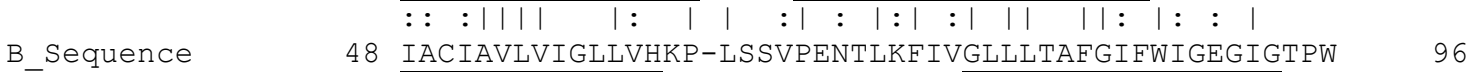

A_Sequence $92----L R A S G F I A L H D E E K A F A S E T D A L A R Q---------\quad 117$

B_Sequence 97 PGEDLSLIGIFAL---LAAFSFIAVRWLRQYHHAQTEPAR 133

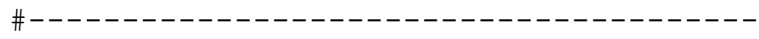

$============$ FINISHED $=============$

Average Quality (AQ) $\quad 22.15$ +/- 7.74

Standard score (Z): 8.0

Precise score (Z): $\mathbf{8 . 0}$ 